\title{
Mobility Modeling of Outdoor Scenarios for MANETs
}

\author{
Illya Stepanov, Pedro José Marrón, and Kurt Rothermel \\ Institute of Parallel and Distributed Systems (IPVS), Universität Stuttgart \\ Universitätsstr. 38, 70569 Stuttgart, Germany \\ \{stepanov,marron,rothermel\}@informatik.uni-stuttgart.de
}

\begin{abstract}
Mobility of users significantly impacts performance of a mobile ad-hoc network. Most existing simulation tools offer only a few random mobility models, which poorly reflect user movements in outdoor scenarios. For example, they do not consider restrictions of a spatial environment. In this paper, we describe a comprehensive and extensible approach to model mobility of users in outdoor scenarios. It reflects the main factors that influence user movement: spatial environments, user travel decisions, and user movement dynamics. We identify model parameters and show how to set them for concrete scenarios. We provide a simulation environment implementing our approach. For concrete scenarios, the environment supports automatic derivation of some parameters from user position traces.
\end{abstract}

\section{Introduction and motivation}

A mobile ad-hoc network (MANET) is composed of mobile devices capable of wireless communication, such as user-carried PDAs and notebooks. These devices cooperate spontaneously without relying on any communication infrastructure.

Many communication protocols and applications for MANETs are under research. Since performing largescale studies in real networks is impractical, simulation tools are often used (see [8], [19], and [35] for details). These tools offer simulation models that represent MANETs and reflect the application behavior, a wireless network protocol stack, the properties of the communication channel (e.g., signal attenuation), and mobility of network clients.

MANETs are frequently intended to be used in outdoor scenarios, e.g., Usenet-on-the-fly [4], CarTALK 2000 [13], and Ad Hoc City [23]. The studies of these scenarios need appropriate simulation support. Regarding the mobility modeling of outdoor scenarios, the existing approaches are neither suitable nor complete. For example, stochastic approaches [9], [12], [14] randomly produce user movements within a rectangular area similar to the Brownian motion of molecular particles. By assuming the simulation area to be free of obstacles, these models do not reflect the spatial environment, which constrains the movements of users. Other approaches focus only on particular characteristics of movement, like places to visit in the graph-based mobility model [40] or movement dynamics in the smooth mobility model [6].

In this paper, we describe a comprehensive approach to model user mobility in outdoor scenarios. It integrates a number of models from different research domains. We also show how to set model parameters for the concrete scenarios and describe how we support automatic derivation of some model parameters from real-world observations, e.g., GPS traces [22]. Our implementation is publicly available and can be downloaded from [11].

The remainder of the paper is structured as follows. Section 2 describes related work in the area of mobility modeling. In Section 3, we present the design of our approach. Sections 4 to 7 describe the components of our model in more detail. In Section 8, we discuss how to set model parameters for concrete scenarios. Section 9 describes our approach for automatic derivation of some model parameters from user position traces. We sum up with a conclusion and an overview of future work in Section 10.

\section{Related work}

Existing approaches for modeling movements of mobile network users can be classified into: random mobility models, area-constrained random models, profile-based models, approaches based on real-world position traces, and integrated models. 
Random mobility models represent user mobility as random movements within a rectangular area, such as the Brownian walk [14], the smooth mobility model [6], and the random waypoint mobility model [9]. These models do not consider constraints of the movement area, and thus poorly reflect real outdoor scenarios.

Area-constrained random models restrict random movements with constraints of a spatial area. Examples are: the random waypoint mobility model with obstacle avoidance [24], the restricted random waypoint mobility model [7], and the graph-based mobility model [40]. Although these models consider spatial environments, they poorly reflect other factors that influence mobility. For example, they do not consider user travel decisions or oversimplify user movement dynamics.

Profile-based models reflect regular travel behavior of a user, e.g., the circle-defined mobility model [28] or the global-local mobility model [29]. These models do not consider spatial environments and use straightforward approaches to reflect user movement dynamics, such as constant-speed movement.

Approaches based on real-world position traces produce user movements according to the traces obtained with a GPS or reconstructed from a trip survey [37]. The traces reflect movements of a limited number of users only, and therefore are not suitable for larger scenarios. Moreover, the traces hardly allow for the variation of parameters like movement speed or the sequence of visited places.

Integrated mobility models reproduce user mobility with a consideration of multiple factors. For example, [30] takes into account constraints of a spatial area, user trips, and user movement profiles. However, the authors are not interested in obtaining complete movement paths. They rather get statistical values for performance analysis of cellular networks. MANET simulation requires a detailed mobility trace. In [31], the authors consider spatial constraints and movement profiles, but use a statistical distribution to reflect user trips. In order to obtain more accurate results, we explicitly model user trips and movement path selection.

It is worth noting that none of the mentioned works correlate its model parameters with real-world observations.

\section{Mobility model design}

In this paper, we aim at constructing a comprehensive, flexible, and scalable mobility model for outdoor scenarios. We base our model on the user- oriented mobility meta-model, which, as shown in [39], is a generic approach to model mobility of users in various simulation scenarios. For outdoor scenarios, we extended the basic model with the modeling of movement path selection and new approaches to reflect user movement dynamics. This allows us to model user mobility more precisely, in particular in city areas.

The user-oriented mobility model considers three key factors that impact user movement in a given area:

- Outdoor environments (movement constraints and points of interest)

- User travel decisions

- User movement dynamics

An outdoor environment constrains movements of users. Network clients move along the certain movement paths like streets and roads, and do not go through obstructions of the movement area. In addition, the outdoor environment contains the socalled "points of interest" (e.g., supermarkets or museums) which normally serve as destination points of movement.

The modeling of user travel decisions includes the modeling of user trip sequences and the modeling of movement path selection. Obviously, people do not move completely random in the target area. According to the activity-based travel demand approach [25], [34], people move to perform an action in certain places, for example, shopping in the particular shops or visiting the predefined sights. A sequence of such actions (trip sequence) predefines user movements in the area. Besides, in order to get to a place where the activity can be executed, a user has the choice among a number of movement paths. Hence, our mobility model also considers the selection of a movement path.

Mobile clients exhibit different movement dynamics. For example, pedestrians tend to move at low speeds with frequent interruptions, while vehicles move at higher speeds and influence dynamics of neighboring vehicles. Since the dynamics of client movement impacts the stability of the network topology, it needs to be reflected in a simulation. To be applied in common MANET scenarios, our implementation supports two major groups of mobile clients: pedestrians and vehicles.

Consequently, the resulting mobility model integrates three sub-models (Fig. 1): spatial model, user trip model, and movement dynamics model.

The spatial model contains a description of the movement area (outdoor environment). It provides the necessary information about the area constraints and the points of interest. The spatial model is initialized from a digital map taken from a geographic information system. 


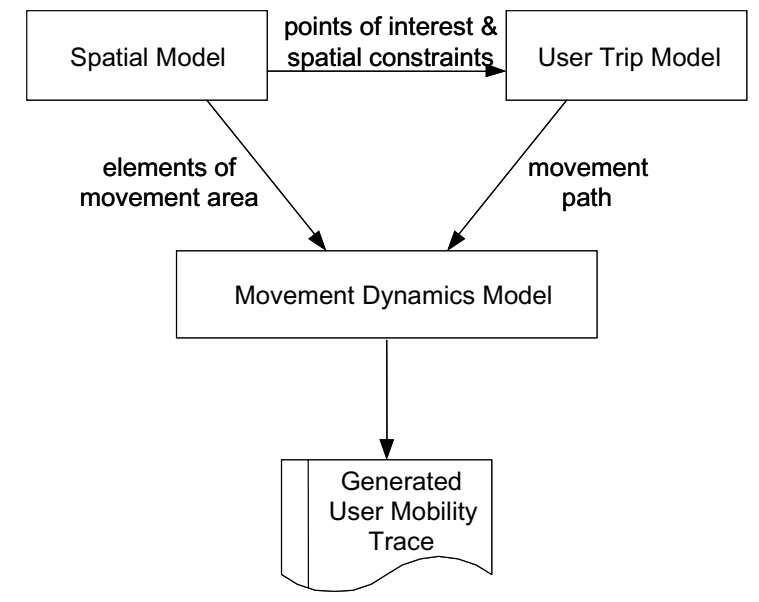

Fig. 1. Structure of the user-oriented mobility model

The user trip model reflects user travel decisions. It performs the mobility modeling at the level of user trips: "Move from point $\mathrm{A}$ to point $\mathrm{B}$ on the path $\mathrm{P}_{\mathrm{AB} 1}$, and then to $\mathrm{C}$ on $\mathrm{P}_{\mathrm{BC} 5}$." The paths chosen for movement reflect the spatial model, i.e. contain points of interest as the movement destination points and consider movement area constraints. To model user trips, we use models from urban transportation planning [32]. The models allow correlation of their parameters with real-world observations.

The movement dynamics model reflects dynamics of user movement (position changes) along their movement paths. Here we use models from physics and vehicular dynamics [36], [43]. The changes of user positions constitute mobility traces, which serve as an input for MANET simulation tools.

The following sections describe the design of these three basic components and their integration.

\section{Spatial model}

The spatial model provides a digital map of the simulation area containing its objects. To standardize the model interface and to be able to use existing map data sources, we have built it according to the Geographic Data Files (GDF) [17] standard.

Thus, as in GDF, the area is represented as the collection of real-world objects, such as streets, museums, hotels, restaurants, cinemas, etc. An object description includes its geometry, attributes, and relations with other objects (Fig. 2). The geometry is specified with primitives, such as points, lines, and polygons. Object attributes contain additional properties, e.g., speed limits or museum opening times. Relations specify meaningful links between the objects

\begin{tabular}{|c|}
\hline general info \\
\hline $\begin{array}{l}\text { id="\#4925" } \\
\text { theme="Road and Ferries" } \\
\text { class="Road Element" }\end{array}$ \\
\hline geometry \\
\hline $\begin{aligned} \text { Line }= & \{(512099.05,5402049.54,0), \\
& (512106.39,5402052.99,0), \\
& \ldots\}\end{aligned}$ \\
\hline attributes \\
\hline $\begin{array}{l}\text { Direction of Traffic Flow="Both } \\
\text { Directions" } \\
\text { Number of Lanes=2 } \\
\text { Average Vehicle Speed }=120 \\
\text { Traffic Jam Sensitivity }=20 \% \\
\text { Length }=5431 \mathrm{~m}\end{array}$ \\
\hline
\end{tabular}

\section{Fig. 2. Example of a road element in GDF}

holding descriptive (e.g., the building belongs to the road) or restrictive (e.g., prohibited maneuver from one road to another) semantics.

GDF specifies some objects as services. Thus, it provides information about the typical activities which people usually perform at the certain points of interest, e.g., shopping at a shopping center, or having lunch in a restaurant. We use this information in the user trip model for trip construction.

To support mobility modeling, we build a topological graph of the movement area (Fig. 3). The vertices represent the locations in the simulation area serving as origins, destinations, or intermediate points of movement. They relate to street crossings and points of interest passed by users, such as monuments, museums, restaurants, and shops. The edges represent the street elements connecting locations. The vertices and the edges are annotated with the properties of the spatial model objects.

In this paper, we focus on mobility modeling for

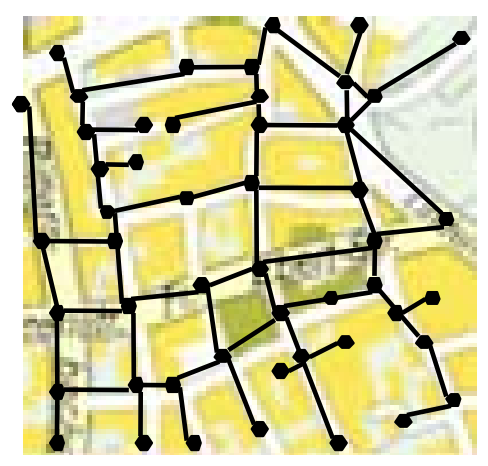

Fig. 3. Representing the spatial model with a topological graph 
MANET outdoor scenarios. We initialize the spatial model from a city map in GDF format. Besides GDF, our implementation also supports maps in other formats, such as the Geometry Markup Language (GML) [18].

\section{User trip model}

The user trip model reflects travel decisions of users. It models the trips users perform during a simulation, and a selection of movement paths. We base this model on the approaches from the domain of urban transportation planning [32].

\subsection{User trips}

In order to define user trips, we use the activitybased travel demand modeling approach [25], [34]. In real life, people do not think about how many trips they are going to perform during a day. Instead, they think about what they want to do, and where these activities can or need to be performed.

As a consequence, we describe user trips with a trip chain. The chain arranges single actions (activities) performed by a user (Fig. 4), such as shopping or sightseeing. It can be defined individually for a user or aggregated over a group of users. For the individually defined trip chain, the sequence of activities is predetermined. The user performs an activity at a corresponding location for some duration of time. The aggregated trip chain accumulates individual trip chains of many users. Hence, users perform activities at multiple locations; different transitions between the activities are possible. Each of the locations and transitions gets a certain "level of popularity" (probability of being selected) reflecting how many users choose the particular transition or location in real life.

The locations for executing activities correspond to

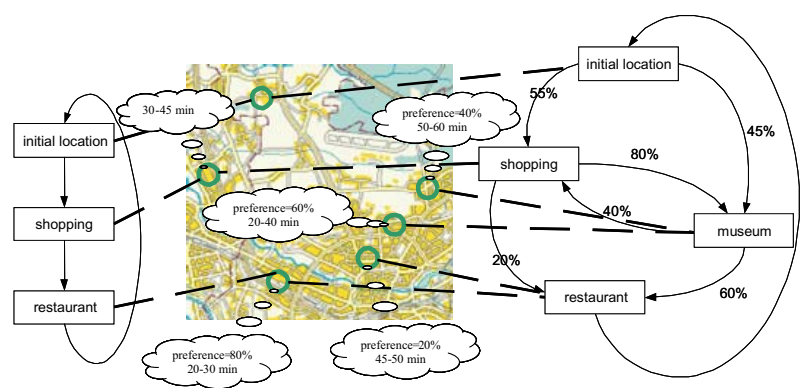

Fig. 4. Example of individual (on the left) and aggregated (on the right) trip chains and their integration with the spatial model points of interest in the spatial model. In a trip chain, they are referenced using either geographic coordinates (e.g., $[1100762.20 \mathrm{~N} ; 4934457.40 \mathrm{E}])$ or symbolic coordinates (e.g., "384 Pitt Street").

During a simulation, mobile users perform activities successively as in their trip chains. In the aggregated chain, the next activity and location for its execution are chosen according to probabilities. The users move from their current position to the location where the next activity is executed. After arriving at the destination, the user stays there for the time needed to execute the activity; then he or she chooses the next activity and starts a new movement.

\subsection{Movement path selection}

To find a movement path between the trip origin and possible destination points, many authors apply simple approaches, like a shortest path algorithm [39], [40]. This poorly reflects real life, since according to investigations in transportation planning, mobile users do not always choose the shortest path for their movement. Diversity in user path selections impacts their mobility in the area and needs to be appropriately reflected in a MANET simulation.

Hence, to model path selection, we use the probabilistic multipath traffic assignment model from transportation planning [15], [32]. The corresponding so-called STOCH algorithm avoids explicit path enumeration. Its computational complexity is comparable to the complexity of the shortest path algorithms. Moreover, the algorithm is based on the multinomial logit model from discrete choice theory [5], [42]. Therefore, the model parameters can be calibrated to fit real-world observations [1], [27].

In the next section, we describe two variations of the algorithm for modeling the path choice of pedestrians and car drivers, and their integration into our mobility model.

\section{Modeling the user path choice}

In the probabilistic multipath traffic assignment model, every path between the trip origin and destination is assigned a selection probability according to the estimated path travel time. Since car drivers and pedestrians estimate travel times differently, we consider two cases.

\subsection{Notation}

We assume a mobile user is currently located at vertex $s$ (trip source vertex) of our spatial model graph 
(Fig. 3). After selecting the next activity, he or she decides to move to vertex $d$ (trip destination vertex). By $e=(i, j)$ we denote a single graph edge directed from vertex $i$ to vertex $j$. The edge represents a transportation link, which is basically a street element from our spatial model. Every edge has the associated cost $t(i, j)$. This cost is the estimated travel time from $i$ to $j$ along the corresponding transportation link. $t^{*}(s, i)$ denotes the shortest cost (the shortest time) to get from $s$ to $i$. Transportation link length is a link's end-to-end travel distance. Link traffic volume $v(e)$ is the number of vehicles currently traveling on the graph edge in the direction from $i$ to $j$.

\subsection{Modeling the path choice of pedestrians}

For pedestrians, we model the path choice as follows. In the first step, we estimate path travel times based on the typical movement speed of the user. In the second step, based on the estimated times, we use the STOCH algorithm [15] to calculate path selection probabilities. In the next paragraphs, we provide a brief description of the algorithm to describe its integration into our mobility model.

For efficiency reasons, the algorithm obviates path enumeration and determines selection probabilities for the individual graph edges. We use these probabilities to select edges for movement successively, thus obtaining the resultant movement path.

The algorithm works with a directed graph. For pedestrians, our spatial model graph is bidirectional and the edge attributes are symmetric.

Similar to user behavior in real life, the algorithm considers only "reasonable" paths with "efficient" links. For the efficient links, the shortest path cost from the trip origin $s$ to the link start vertex $i$ is less than the shortest path cost from $s$ to the link end vertex $j$ (thus, users would come farther from the origin with every move):

$$
\forall e=(i, j): t^{*}(s, i)<t^{*}(s, j)
$$

This reduces the number of links under consideration. The algorithm assumes that the selection probability $a$ for link $e=(i, j)$ is exponentially dependant on the difference between the cost of the shortest path from $s$ to $j$ and the cost of the shortest path from $s$ to $j$ that contains the link $e$ :

$$
a(e)=e^{\theta\left[t^{*}(s, j)-t^{*}(s, i)-t(i, j)\right]}
$$

The parameter $\theta(\theta \geq 0)$ is set by the modeler. It reflects the importance of the path length for the user choice and user knowledge about the movement area. As $\theta$ increases (i.e., the path length becomes more important and the user knows that the particular paths are shorter), the selection probabilities for shorter paths also increase. When $\theta$ is zero (i.e., the path length is not important or the user cannot estimate it), all efficient paths are considered equally. The exponential expression is analogous to the expression of choice probability from the multinomial logit discrete choice model. Thus, the parameter $\theta$ can be correlated with real-world observations by maximizing the so-called log-likelihood function using methods of numerical maximization, as described in [42].

The algorithm performs three steps (Fig. 5). During the first step ("Initialization"), it calculates the shortest path costs from $s$ to all other vertices. They are used to estimate the link selection likelihoods $a(e)$. In this step, the non-efficient links are assigned a likelihood of zero, and thus are excluded from further consideration.

\section{Initialization}

1. Calculate shortest path costs $t^{*}$ from the trip origin $s$ to all other graph vertices using a shortest path algorithm (e.g., Dijkstra [16]).

2. For each edge $e=(i, j)$, determine its likelihood $a(e)$ :

$$
a(e)= \begin{cases}e^{\theta\left[t^{*}(s, j)-t^{*}(s, i)-t(i, j)\right]}, & \text { if } t^{*}(s, i)<t^{*}(s, j) \\ 0, & \text { otherwise }\end{cases}
$$

\section{Forward Step}

Starting with the origin $s$, for each edge $e=(i, j)$ determine its weight (likelihood with respect to likelihoods of the edges having $i$ as end vertex (denoted as $\left.F_{i}\right)$ ):

$$
w(e)= \begin{cases}a(e), & \text { if } F_{i}=\varnothing \\ a(e) \sum_{e^{\prime} \text { in } F_{i}} w\left(e^{\prime}\right), & \text { otherwise }\end{cases}
$$

Stop when the destination $d$ is reached.

\section{Backward Step}

Starting from the destination $d$, for each edge $e=(i, j)$ determine its conditional selection probability $p(e \mid j)$ with respect to the edges having $j$ as end vertex (denoted as $F_{j}$ ):

$$
p(e \mid j)=\frac{w(e)}{\sum_{e^{\prime} \text { in } F_{j}} w\left(e^{\prime}\right)}
$$

Stop when the origin $\mathrm{s}$ is reached.

Fig. 5. Algorithm to estimate conditional
edge selection probabilities (STOCH
algorithm)


During the next step ("Forward Step"), the algorithm computes link weights $w(e)$ depending on the weights of the topologically preceding links. In the third step ("Backward Step"), the algorithm estimates conditional link selection probabilities $p(e \mid j)$. Obviously, the sum of the conditional selection probabilities of edges having the same end vertex equals 1 .

It is important to note that the third step of the presented algorithm differs slightly from the third step of the original STOCH algorithm. Since the goal of the original algorithm is to distribute a number of moving users among the transportation links, it assigns link traffic volumes. In this paper, we calculate the link selection probabilities.

It is clear that the algorithm terminates during the "forward" and "backward" steps. Since it excludes the non-efficient links from consideration, it traverses the graph edges successively in the topological order, until it reaches the trip destination vertex $d$.

An example of using the STOCH algorithm to calculate link selection probabilities is depicted in Fig. 6. The example shows a subset of the spatial model graph that contains efficient links for the trip from the vertex $s$ to the vertex $d$. Based on the estimated travel times (posted above the edges), conditional link selection probabilities are obtained (posted below the edges). They provide the possibility that the link and its end vertex are visited. In the example, we use $\theta=0.9$. Having the probabilities calculated, it is a straightforward task to traverse the graph in the reverse direction from the destination to the source, and to choose edges stochastically at each intermediate vertex, thus constructing the selected movement path.

Following this method, a path choice for pedestrians is performed as follows:

1. Estimate conditional selection probabilities for the efficient graph edges using the STOCH algorithm.

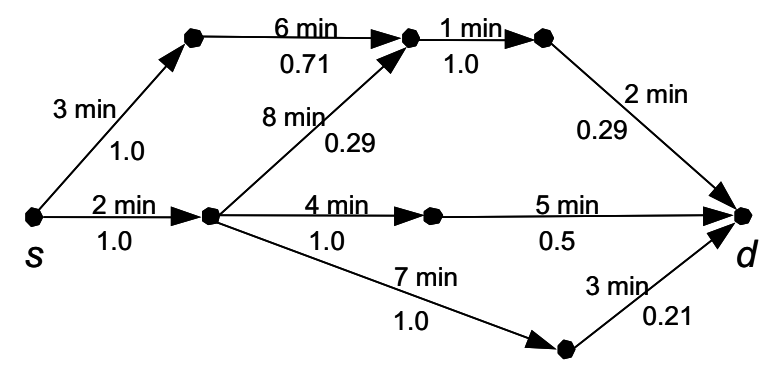

Fig. 6. Example of calculating link selection probabilities using the STOCH algorithm
2. Decide on a movement path by traversing the graph in the reverse direction from the destination vertex to the source vertex. The resultant path is successively constructed by adding edges. The edges are chosen stochastically at each intermediate vertex from the set of efficient incoming edges in accordance with the computed selection probabilities (i.e. the edge with higher selection probability has a better chance of being selected).

Since, according to our model, link travel times for a pedestrian depend only on link end-to-end distances, the corresponding edge costs do not change for the user over time. Hence, the STOCH algorithm needs to be applied only once for this user and for the given source and destination vertices. Once computed, the probabilities are reused by successive calculations.

\subsection{Modeling the path choice of car drivers}

For car drivers, travel time on a transportation link depends not only on the link length, but also on the current traffic volume. Travel time is longer on congested roads, so drivers try to avoid them.

In transportation science, the so-called "volumedelay" functions model the impact of the link traffic volume on the link travel time (its cost), e.g., the Bureau of Public Roads (BPR) function [10], the Overgaard function [33], and the Spiess function [38]. Although we could use any of these functions, we choose the BPR function, because it is well investigated and its parameters for different road types are well documented (see [21] for details).

The BPR function defines the following dependency between the link travel time $t$ and the link traffic volume $Q$ :

$$
t(Q)=t_{0}\left[1+\alpha\left(\frac{Q}{Q_{\max }}\right)^{\beta}\right]
$$

where $t_{0}$ is the link travel time at free traffic flow. It is estimated from the typical vehicle travel speed and the link length.

$\alpha$ and $\beta$ are empirical coefficients. Their values for the concrete road classes are available. Our implementation relies on the standard values $(\alpha=0.15$ and $\beta=4)$ and on the values from [21] with the spatial model providing the necessary road class identifiers.

$Q$ is the current link traffic volume. It is calculated from the current density of vehicles (veh./m) traveling on a graph edge in the direction from the start vertex to the end vertex, and the average traffic speed. 
$Q_{\max }$ is the maximum link capacity. We rely on the typical link capacity values from [3] and [26].

We use the BPR function to calculate estimated link travel times for vehicles. Then we apply the STOCH algorithm to calculate link selection probabilities. Upon calculating the probabilities, we also consider whether a movement is prohibited for vehicles along a particular road element or in a particular direction. In this case, the element is assigned the probability $p(e \mid j)=0$.

To sum-up, the path choice for car drivers is made as follows:

1. Calculate the link costs based on the current link traffic flows using the BPR function (3).

2. Estimate conditional selection probabilities for efficient edges using the STOCH algorithm.

3. Decide on a movement path by traversing the graph in the reverse direction from the destination vertex to the source vertex. The resulting path is successively constructed by adding edges. The edges are stochastically chosen at each intermediate vertex from the set of efficient incoming edges in accordance with the computed selection probabilities.

As opposed to the pedestrian case, link travel times change dynamically due to changing traffic volumes. Therefore, we need to compute selection probabilities each time upon simulating a selection of a movement path for a user.

\subsection{Possible extensions for the path selection modeling}

An interesting option would be to apply the STOCH algorithm in the opposite direction from the trip destination to the trip source point. As the result, we obtain the conditional link selection probability providing the possibility that the link and its start vertex are visited. This allows deciding on a movement path by traversing the graph starting from the source vertex. According to [41], it reflects user behavior better in some cases.

It is also possible to model user path choices considering factors other than the estimated travel time, e.g., the number of sights passed or the number of stop signs. In this case, a modeler defines a socalled "behavioral process" function [42]. The function is a linear combination of attributes impacting the user choice. It is estimated by a modeler for a specific situation and for a specific group of users. In future work, we plan to support the behavioral process, which is defined using attributes of spatial model objects.

\section{Movement dynamics model}

The movement dynamics model defines patterns in speed and direction changes of mobile clients during their movement between two locations. It is based on models from physics and vehicular dynamics.

Different dynamics models are proposed for different types of mobile clients. For example, the lowspeed motion along road edges with frequent stop-andgo behavior reflects the dynamics of pedestrians. There are models for vehicles based on correlations of speed and direction changes (e.g., smooth random mobility model [6]), approaches from traffic modeling (e.g., fluid traffic model [36]), and dependencies between movements of neighboring vehicles (e.g., intelligent driver model [43]). In addition, we can integrate special models for the certain aspects of movement dynamics, such as lane change behavior [2]. To improve the accuracy of modeling, the movement dynamics model can also consider the attributes of road elements, such as the speed limit or the number of traffic lanes.

Our present implementation includes a number of movement dynamics models for pedestrians and vehicles. We find the following models the most interesting for the outdoor scenarios.

\subsection{Modeling the dynamics of pedestrians}

We model the dynamics of pedestrians as a constant speed motion. The speed is randomly chosen at the beginning of the movement from a certain interval. For example, for pedestrians walking freely in a city, typical values are between 4 and $5 \mathrm{~km} / \mathrm{h}$ [26].

\subsection{Modeling the dynamics of vehicles}

For modeling the dynamics of vehicles, we use the intelligent driver model described in [43]. According to the model, vehicles try to keep certain distance between each other for their safety. The acceleration of vehicle $i$ at time $t$ depends on its current velocity $v_{i}(t)$, the distance $s_{i, i-1}(t)$ to the vehicle $i-1$ in front, and the safety distance $s_{i, i-1}^{*}(t)$ between the vehicles:

$$
\dot{v}_{i}=a_{i}\left(1-\left(\frac{v_{i}}{v_{i}^{0}}\right)^{\sigma}-\left(\frac{s_{i, i-1}^{*}}{s_{i, i-1}}\right)^{2}\right)
$$

In the formula, $a_{i}$ is the maximum acceleration of the vehicle $i$.

$v_{i}^{0}$ is the desired speed of vehicle $i$ which depends on the behavior of the driver. In our simulations, we choose randomly the desired speed for a vehicle from a 
certain interval, e.g., between $40 \mathrm{~km} / \mathrm{h}$ and $50 \mathrm{~km} / \mathrm{h}$ in city scenarios.

$\sigma$ is the exponent controlling the acceleration behavior of vehicles until they reach their desired speed.

The safety distance $s^{*}{ }_{i, i-1}(t)$ between vehicles $i$ and $i+1$ is expressed as:

$$
s_{i, i-1}^{*}=s_{0}+\max \left(v_{i} T_{i}+\frac{v_{i} \Delta v_{i, i-1}}{2 \sqrt{a_{i} b_{i}}}, 0\right)
$$

where $s_{0}$ is the minimum distance between vehicles, like in a traffic jam.

$T_{i}$ is the safe time headway in congested traffic. It represents the maximum reaction time of a driver, e.g., to apply breaks.

$\Delta v_{i, i-1}$ is the difference in velocities between the vehicles $i$ and $i-1: \Delta v_{i, i-1}=v_{i}-v_{i-1}$.

$b_{i}$ is the driver's typical deceleration in regular (e.g., non-critical) situations.

According to the model, on distances $s_{i, i-1}>s_{i, i-1}{ }^{*}$ the vehicle $i$ accelerates until it reaches its desired speed. As the gap $s_{i, i-1}$ approaches the safety distance, the vehicle decreases its acceleration. When $s_{i, i-1} \leq s_{i, i-1}{ }^{*}$, the vehicle breaks harder to avoid a collision.

The model can handle traffic jam situations, e.g., as the leading vehicles slow down, the succeeding vehicles will also decrease their speeds.

Typical values of coefficients as described in [43] are: $a_{i}=0.6 \mathrm{~m} / \mathrm{s}^{2}, \sigma=4, T_{i}=1.5 \mathrm{~s}, s_{0}=2 \mathrm{~m}, l_{i}=5 \mathrm{~m}$, and $b_{i}=0.9 \mathrm{~m} / \mathrm{s}^{2}$. Our implementation uses these values by default.

The described model does not explicitly reflect movement on multiple traffic lanes. The reason is that in our road data each lane is represented as a separate road element, hence the multilane traffic is still reflected.

By integrating the described model into our simulation environment we make it useful for performance evaluations of vehicular ad-hoc networks (VANETs). To the best of our knowledge, no other freeware tool currently exists to generate VANET mobility traces for MANET simulation tools.

\section{Defining model parameters}

In order to use the described approach in simulations, it is necessary to define the spatial model, user trip model, and movement dynamics model. They can be defined according to the simulation scenario for every user individually or for a group of users.

The spatial model contains a digital map of the simulation area. Since we implemented parsers for
GDF and GML, its initialization from the given geographic information system is straightforward.

The parameters of movement dynamics are specific to a particular model. For the models described in this paper, either the model authors specify typical values (e.g., for the intelligent driver model) or they can be easily estimated based upon the daily experiences.

Parameters of the user trip model include trip chains with activities, places for executing activities, times needed to execute activities, transitions between activities, and the parameter $\theta$ for path selection. Defining the user trip model requires more effort. To make things simpler, our implementation provides random generation of user trips. The path selection modeling can also be simplified, for example, by setting $\theta$ to 0 which makes mobile users consider all efficient paths equally. Setting $\theta$ to some large value makes the users select only the shortest paths. In these two cases, our path selection model performs as a multiple-path searching algorithm.

To simulate mobility in concrete scenarios more accurately, our implementation also provides automatic derivation of trip model parameters from position traces, e.g., GPS traces, as described in the next section. One might argue that if we already have position traces, it is sufficient to simulate mobility according to them. However, the trace represents only a limited number of clients. Having the model parameters defined, it is possible to simulate any number of mobile clients. In addition, the model parameters can be varied to check their effect on simulation results.

\section{Deriving user trip model parameters from position traces}

Our approach is quite simple and is based on associating the locations in the area with the typical activities. A more sophisticated approach is based on heuristic and is described in [44].

We consider a client position trace as a sequence of entries having the following form:

- Client ID

- Time

- Client position (coordinate)

The entries are stored at constant intervals. The authors in [44] state that in order to reconstruct movement paths for vehicles, the granularity should be less than $10 \mathrm{~s}$. For pedestrians, the granularity can be estimated proportionally.

For constructing an aggregated trip chain from the traces, we perform the following steps. 


\subsection{Associate activities with locations}

Using information from the spatial model ("theme" and "class code" of spatial model objects), we associate activities with corresponding locations in the target area. This allows the "reverse" mapping from a visited point to the performed activity. Thus, if we detect a user stays at a particular location, we can also determine which activity he or she performed there.

The association is unambiguous, i.e. although multiple activities can be performed at a point, we consider only the primary activity (e.g., a shopping center is used only for shopping).

Our current implementation automatically distinguishes the following activities provided by GDF data sources: "business", "cultural", "educational", "meal", "parking", "recreation", "shopping", and "sightseeing". Besides, we introduce two special activities: "initial" and "unclassified". The initial positions of mobile users in the trace sample are associated with the "initial" activity. All the points of interest that do not belong to one of the standard activities are associated with the "unclassified" activity. Moreover, if upon a trace analysis we detect a user stays at a location, which is not associated with an activity, it is also added to the "unclassified" activity.

Obviously, the described splitting into activities is neither obligatory nor complete. It just reflects our current simulation scenarios. In our implementation, it is possible to change the standard associations and / or introduce new ones.

\subsection{Determine the trip chain parameters}

The parameters to be determined are: probabilities of transitions between activities, visiting probabilities of locations, and durations of activity executions at the locations. These parameters are obtained by comparing client positions with coordinates of points of interest. Thus, we process trace entries of every user in chronological order and check the following:

- If a client stays within a point of interest longer than a threshold value (minimal activity execution time), we then detect an activity by:

- Finding the corresponding activity;

- Incrementing the counter for this activity;

- Incrementing the counter for this activity at this location;

- Incrementing the number of transitions between the previous activity and the current activity.

- If a client departs from a point of interest (i.e. his or her position is no longer within the previous point of interest):

- Update durations of executing the activity at this location (in our implementation, we obtain the minimal and maximal values (Fig. 4); as an option, we could also use the average or the weighted average).

After the trace data is processed completely, we calculate the selection probabilities for locations within every activity (location's preference) by dividing the counter for the activity at the location by the counter for the activity. We calculate the probabilities of transitions between two activities by dividing the count of transitions between the activities by the total count of transitions from the source activity.

It is important to note that by applying the described method for vehicles, we primarily detect the "parking" activity. To further differentiate between activities, we must additionally process movements of car drivers after parking.

\subsection{Determine the parameter $\theta$ of path selection model}

To determine this parameter, we need to count how many times a particular transportation link is used for a trip between two given locations. Then $\theta$ needs to be calibrated for each pair of vertices between which the trips are performed. For calibration, a method of numerical maximization (e.g., the method of steepest ascent) can be used, as described in [42]. Then the single value for the system can be calculated as the weighted average of the calibrated $\theta_{i}$.

For vehicles, we also need to consider traffic volumes on transportation links, since they impact link costs in our model. So far, we do not provide the calibration of $\theta$ in our prototype, but its implementation is planned exactly as described.

\section{Conclusion}

Many authors have shown that user mobility has a significant impact on the performance of MANETs [9], [12], [24], [40]. Hence, it needs to be appropriately reflected in simulations. In this paper, we have focused on mobility modeling in outdoor scenarios and proposed a comprehensive approach. Our approach considers a geographic model of the simulation area, user trip sequences with path selection decisions, and user movement dynamics. We have implemented it in our publicly available framework for mobility modeling [11]. It is a stand-alone application which produces mobility traces for various MANET 
simulation or emulation tools, such as NS-2 [8], GloMoSim [19], or Network Emulation Testbed [20].

We made our implementation easy-to-use. Hence, it supports processing of digital maps in common formats, random generation of user trips in the area, and a number of movement dynamics models for pedestrians and vehicles with typical parameters. Obviously, the random generation of user trips is only introduced to minimize the model creation overhead. To produce user trips more accurately, the framework can also derive trip model parameters from actual position traces.

In future work, we plan on using our approach in simulations and emulations of MANET outdoor scenarios in order to obtain more accurate evaluation results.

\section{Acknowledgment}

Illya Stepanov is funded by the Landesgraduiertenförderung of the state of BadenWürttemberg (graduate funding for $\mathrm{PhD}$ studies).

We want to thank Martin Bauer, Jan Geiger, Timo Heiber, Daniel Herrscher, Kateryna Ignatova, Abdelmajid Khelil, Andreas Lachenmann, Steffen Maier, and Christoph Ruffner for reading a draft of the paper and for their valuable suggestions.

Illya Stepanov is $\mathrm{PhD}$ student at both the Universität Stuttgart and the Donetsk National Technical University, Ukraine. We thank Prof. Svyatnyj from the Donetsk National Technical University, Ukraine for his cooperation.

\section{References}

[1] J. E. Abraham, "Parameter Estimation in Urban Models: Theory and Application to a Land Use Transport Interaction Model of the Sacramento, California Region," PhD Thesis, Department of Civil Engineering, University of Calgary, Canada, January 2000.

[2] K. I. Ahmed, "Modeling Drivers' Acceleration and Lane Changing Behavior," PhD Thesis, Massachusetts Institute of Technology, February 1999.

[3] R. Akçelik, "Travel time functions for transport planning purposes: Davidson's function, its timedependent form and an alternative travel time function", Australian Road Research 21 (3), pp. 49-59, 1991.

[4] C. Becker, M. Bauer, J. Hähner, "Usenet-on-the-fly: supporting locality of information in spontaneous networking environments," in Proceedings of CSCW 2002 Workshop on Ad hoc Communications and Collaboration in Ubiquitous Computing Environments, New Orleans, USA, 2002.
[5] M. Ben-Akiva, M. Bierlaire, "Discrete choice methods and their applications to short-term travel decisions," in R. Hall (ed.), Handbook of Transportation Science, International Series in Operations Research and Management Science, Vol. 23, Kluwer, 1999.

[6] C. Bettstetter, "Smooth is Better than Sharp: A Random Mobility Model for Simulation of Wireless Networks," in Proceedings of the 4th ACM International Workshop on Modeling, Analysis, and Simulation of Wireless and Mobile Systems (MSWiM'01), Rome, Italy, July 2001.

[7] L. Blazevic, S. Giordano, J. Y. Le Boudec, "Self Organized Terminode Routing," Technical Report No. DSC/2000/040, Swiss Federal Institute of Technology, Lausanne, December 2000.

[8] L. Breslau, D. Estrin, K. Fall, S. Floyd, J. Heidemann, A. Helmy, P. Huang, S. McCanne, K. Varadhan, Y. $\mathrm{Xu}$, and $\mathrm{H}$. Yu, "Advances in Network Simulation," IEEE Computer, Vol. 33, No. 5, pp. 59-67, May 2000.

[9] J. Broch, D. A. Maltz, D. B. Johnson, Y.-C. Hu, J. Jetcheva, "A Performance Comparison of Multi-Hop Wireless Ad Hoc Network Routing Protocols," in Proceedings of the 4th Annual ACM/IEEE International Conference on Mobile Computing and Networking, Dallas, TX, October 1998.

[10] Bureau of Public Roads, Traffic Assignment Manual, U.S. Dept. of Commerce, Urban Planning Division, Washington D.C., 1964.

[11] CANU Mobility Simulation Environment (CanuMobiSim) Home Page. Available at http://canu.informatik.uni-stuttgart.de/mobisim

[12] T. Camp, J. Boleng, V. Davies, "A Survey of Mobility Models for Ad Hoc Network Research," Wireless Communication \& Mobile Computing (WCMC): Special issue on Mobile Ad Hoc Networking: Research, Trends and Applications, vol. 2, no. 5, pp. 483-502, 2002.

[13] CarTalk 2000 Project Home Page. Available at http://www.cartalk2000.net

[14] S. R. Das, R. Castaneda, J. Yan, R. Sengupta, "Comparative performance evaluation of routing protocols for mobile ad hoc networks," in Proceedings of the 7th International Conference on Computer Communications and Networks (ICCCN), pp. 153-161, October 1998.

[15] R. B. Dial, "A probabilistic multipath traffic assignment model which obviates path enumeration," Transportation Research, No. 5, 1971.

[16] E. W. Dijkstra, "A note on two problems in connection with graphs," Numerische Mathematik, 1, pp. 269-271, 1959.

[17] Geographic Data Files (GDF) Home Page. Available at http://www.ertico.com/links/gdf/gdf.htm

[18] Geometry Markup Language (GML) 2.0, OpenGIS ${ }^{\circledR}$ Implementation Specification, OGC Document Number: 01-029, 20 February 2001. Available at http://www.opengis.net/gml/01-029/GML2.html 
[19] GloMoSim: Global Mobile Information Systems Simulation Library. Available at http://pcl.cs.ucla.edu/projects/glomosim/

[20] D. Herrscher, K. Rothermel, "A Dynamic Network Scenario Emulation Tool", in Proceedings of the 11th International Conference on Computer Communications and Networks (ICCCN), Miami, October 2002.

[21] A. J. Horowitz, "Delay-Volume Relations for Travel Forecasting: Based on the 1985 Highway Capacity Manual," Department of Civil Engineering and Mechanics University of Wisconsin - Milwaukee, March 1991.

[22] O. Jan, A. J. Horowitz, Z.-R. Peng, "Using GPS Data to Understand Variations in Path Choice," Transportation Research Record Journal, Vol. 1725, 2000.

[23] J. G. Jetcheva, Y.-C. Hu, S. PalChaudhuri, A. K. Saha, D. B. Johnson, "Design and Evaluation of a Metropolitan Area Multitier Wireless Ad Hoc Network Architecture," in Proceedings of the 5th IEEE Workshop on Mobile Computing Systems \& Applications, Monterey, California, October 09-10, 2003.

[24] P. Johanson, T. Larsson, N. Hedman, B. Mielczarek, and M. Degermark, "Scenario-based Performance Analysis of Routing Protocols for Mobile Ad-hoc Networks," in Proceedings of ACM/IEEE MOBICOM'99, pp. 195-206, Seattle, WA, August 1999.

[25] R. Kitamura, "Applications of Models of Activity Behavior for Activity Based Demand Forecasting," in Proceedings of Activity-Based Travel Forecasting Conference, June 1996.

[26] C. J. Khisty, Transportation Engineering: An Introduction, Prentice Hall, 1990.

[27] M.-T. Li, "Asymmetrically-Distributed TravelerPerceived Errors in the Travel Time Disutility Function for Stochastic User-Equilibrium Traffic Assignment," $\mathrm{PhD}$ Thesis, Department of Civil Engineering, University of Florida, USA, December, 1999.

[28] G. Liu and G. Maguire, "Efficient Mobility Management Support for Wireless Data Services," in Proceedings of the 45th IEEE Vehicular Technology Conference (VTC'95), Chicago, Illinois, USA, July 2628, 1995

[29] T. Liu, P. Bahl, "Mobility Modeling, Location Tracking, and Trajectory Prediction in Wireless ATM Networks," IEEE Journal on Special Areas in Communications, Special Issue on Wireless Access Broadband Networks, Vol. 16, No. 6, August 1998.

[30] J.G. Markoulidakis, G.L. Lyberopoulos, D.F. Tsirkas, E.D. Sykas, "Mobility Modeling in Third-Generation Mobile Telecommunications Systems," IEEE Personal Communications, pp. 41-56, August 1997.
[31] S. Nousiainen, K. Kordybach, P. Kemppi, "User Distribution and Mobility Model Framework for Cellular Network Simulations," in Proceedings of IST Mobile \& Wireless Telecommunications Summit 2002, Thessaloniki, Greece, June 2002.

[32] N. Oppenheim, Urban Travel Demand Modeling: From Individual Choices to General Equilibrium, Wiley-Interscience, January 1995.

[33] K. R. Overgaard, "Urban Transportation Planning: Traffic Estimation", Traffic Quarterly, 1967.

[34] E. I. Pas, "Recent Advances in Activity-Based Travel Demand Modeling," in Proceedings of Activity-Based Travel Forecasting Conference, June 1996.

[35] G. Riley, "The Georgia Tech Network Simulator," in Proceedings of the ACM SIGCOMM workshop on Models, Methods and Tools for Reproducible Network Research (MoMeTools), Karlsruhe, Germany, August 2003.

[36] I. Seskar, S. V. Marie, J. Holtzman, J. Wasserman, "Rate of Location Area Updates in Cellular Systems," in Proceedings of IEEE VTC'92, Denver, CO, May 1992.

[37] J. Scourias, T. Kunz, "An activity-based mobility model and location management simulation framework," in Proceedings of the Second ACM International Workshop on Modeling and Simulation of Wireless and Mobile Systems (MSWiM'99), Seattle, USA, August 1999.

[38] H. Spiess, "Conical Volume-Delay Functions," Transportation Science, Vol. 24, No. 2, 1990.

[39] I. Stepanov, J. Haehner, C. Becker, J. Tian, K. Rothermel, "A Meta-Model and Framework for User Mobility in Mobile Networks," in Proceedings of 11th IEEE International Conference on Networks 2003 (ICON'2003), Sydney, Australia, October 2003.

[40] J. Tian, J. Hähner, C. Becker, I. Stepanov, K. Rothermel, "Graph-based Mobility Model for Mobile Ad Hoc Network Simulation," in Proceedings of the 35th Annual Simulation Symposium, San Diego, California, April 2002.

[41] R. Tobin, "An Extension of Dial's Algorithm Utilizing a Model of Tripmakers' Perceptions," Transportation Research, Vol. 11, 1977.

[42] K. Train, Discrete Choice Methods with Simulation, Cambridge University Press, January 2003.

[43] M. Treiber, D. Helbing, "Explanation of Observed Features of Self-Organization in Traffic Flow," Preprint cond-mat/9901239 (1999).

[44] J. Wolf, S. Schönfelder, U. Samaga, M. Oliveira, K. W. Axhausen, "Eighty Weeks of GPS Traces: Approaches to Enriching Trip Information," in Proceedings of the 83rd Annual Meeting of the Transportation Research Board, Washington D.C., January 2003. 